UCRL-CR-125216

S/C- 923-003

\title{
Precise Measurements of Hyperfine Components in the Spectrum of Molecular Iodine
}

\author{
C. J. Sansonetti
}

May 23, 1996

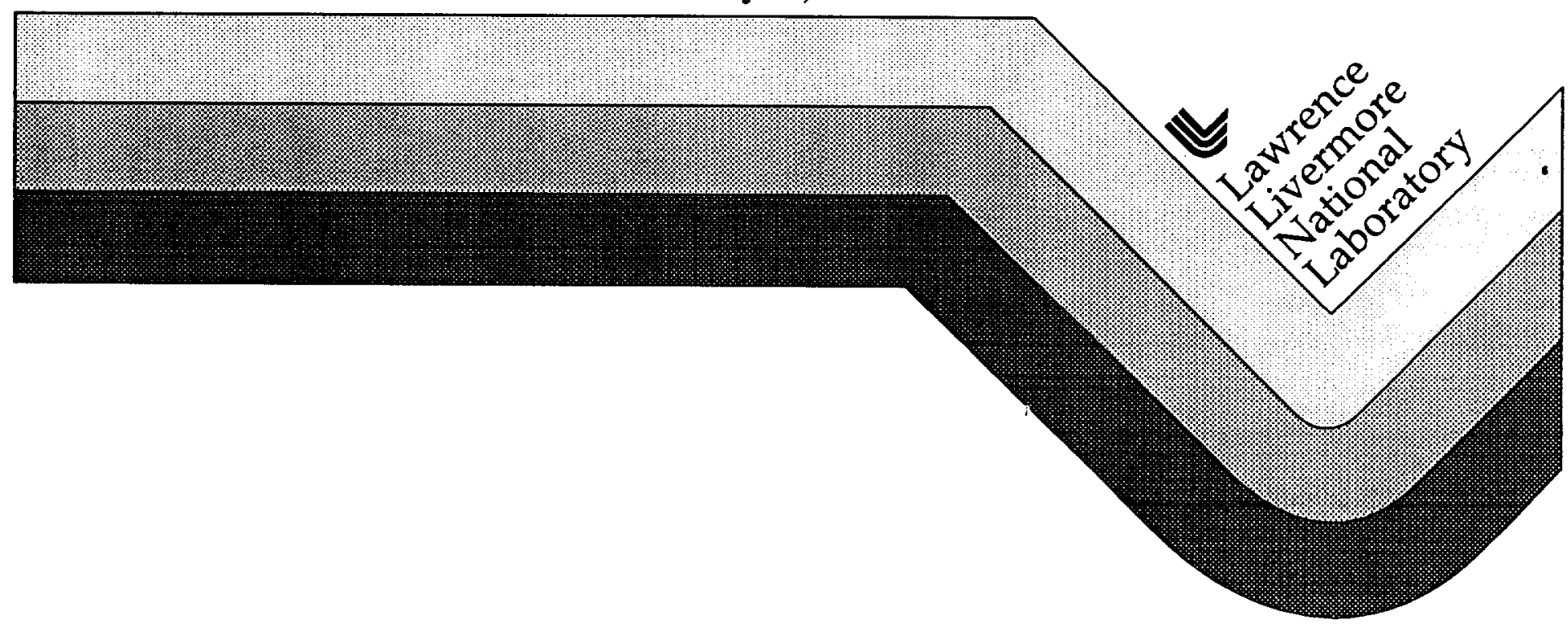




\section{DISCLAIMER}

This document was prepared as an account of work sponsored by an agency of the United States Government. Neither the United States Government nor the University of California nor any of their employees, makes any warranty, express or implied, or assumes any legal liability or responsibility for the accuracy, completeness, or usefulness of any information, apparatus, product, or process disclosed, or represents that its use would not infringe privately owned rights. Reference herein to any specific commercial product, process, or service by trade name, trademark, manufacturer, or otherwise, does not necessarily constitute or imply its endorsement, recommendation, or favoring by the United States Government or the University of California. The views and opinions of authors expressed herein do not necessarily state or reflect those of the United States Government or the University of California, and shall not be used for advertising or product endorsement purposes.

Work performed under the auspices of the U.S. Department of Energy by Lawrence Livermore National Laboratory under Contract W-7405-ENG-48. 


\author{
Precise Measurements of Hyperfine Components \\ in the Spectrum of Molecular Iodine
}

Craig J. Sansonetti

\author{
National Institute of Standards and Technology \\ Gaithersburg, MD 20899
}

May 23, 1996

\begin{abstract}
Absolute wave numbers with a typical uncertainty of $1 \mathrm{MHz}$ (95\% confidence) have been measured for 102 hyperfine-structure components of ${ }^{127} \mathrm{I}_{2}$. The data cover the range 560 tc $656 \mathrm{~nm}$ with no gaps larger than $50 \mathrm{~cm}^{-1}$. The spectra were observed by using Doppler-free frequency modulation spectroscopy with a tunable $\mathrm{cw}$ laser. The laser was locked to selected iodine components, and its wave number was measured with a high precision Fabry-Perot wavemeter. The accuracy of the results is confirmed by the good agreement of 9 of the lines with previous results from other laboratories. These measurements provide a welldistributed set of precise reference lines for this spectral region.
\end{abstract}




\section{INTRODUCTION}

Absorption lines of molecular iodine $\left(I_{2}\right)$ provide one of the most convenient sources of wavelength standards for laser spectroscopy. The entire range of the $I_{2}$ B-X band system has been studied at high resolution by Fourier transform spectroscopy and presented in an atlas by Gerstenkorn and Luc. ${ }^{1}$ With a small correction presented in a later paper, ${ }^{2}$ the atlas wave numbers provide a dense comb of standards covering the green to red portions of the spectrum with an accuracy of about $0.002 \mathrm{~cm}^{-1}$. This relative accuracy, about a part in $10^{7}$, is satisfactory for calibration of spectra recorded with broadband lasers or by classical methods. But for precise measurements with stable single-frequency lasers, standards of higher accuracy are needed.

The Doppler-limited $I_{2}$ absorption spectrum is not suitable for use as a standard at higher accuracy because its line profiles have varying asymmetries that reflect underlying hyperfine structure. Each iodine line is a composite of 15 or 21 hyperfine components that cannot be resolved using classical methods. By Doppler-free laser spectroscopy, however, these components can be fully resolved and used as well-defined reference lines. Notable cases in point are the lines used to stabilize He-Ne lasers at 633 and $612 \mathrm{~nm}$ and the argon ion laser at $514 \mathrm{~nm}^{3}$

A number of groups have made precise measurements of selected iodine lines for calibration purposes in particular experiments. The largest group of lines (27) was reported by Hlousek and Fairbank ${ }^{4}$ in connection with their measurements of Doppler-free two-photon and saturated-absorption lines in helium. Most of their iodine lines, however, were measured with only modest accuracy $\left(0.0002 \mathrm{~cm}^{-1}\right)$. A few lines have been reported by other groups with much higher accuracy. $5,6,7$ 
Over the past decade hyperfine components in $102 \mathrm{I}_{2}$ lines spanning the range from 560 to $656 \mathrm{~nm}$ have been measured in our laboratory at NIST. The accuracy of the results is generally about 2 parts in $10^{9}$. Coverage of this spectral region (15233 to $17845 \mathrm{~cm}^{-1}$ ) is fairly uniform. There are no gaps wider than $50 \mathrm{~cm}^{-1}$. In this paper we present all of our Doppler-free iodine measurements. The results should be useful to other workers who need high-accuracy reference wavelengths in this spectral region.

\section{EXPERIMENT}

Because this paper represents a collection of data measured in a number of experiments over a period of nearly ten years, not all of the measurements were made under identical experimental conditions. Almost all of the observations reported here have been made using Doppler-free frequency modulation (FM) spectroscopy, although some of the earliest measurements employed a simple saturated absorption setup with a frequency dither imposed on the tunable dye laser for locking to the iodine lines. Since the observed wave numbers do not depend on the exact Doppler-free technique employed, we will not discuss the variations in technique that may apply to some of the measurements. The experimental appartus and methods described here are representative of those used for most of the data.

Our technique for observing Doppler-free spectra of $\mathrm{I}_{2}$ lines by FM spectroscopy is essentially similar to that described by Hall, Holberg, Baer, and Robinson. ${ }^{8}$ The theory of FM spectroscopy including a detailed analysis of the observed line shapes has been presented by Bjorklund, Levenson, Lenth, and Ortiz. ${ }^{9}$ 
Apparatus typical of that used in these measurements is shown in Figs. 1 and 2. A commercial ring dye laser operated with the dyes Rhodamine $6 G, D C M$, or Rhodamine 110 provides tunable single-frequency radiation with a line width of less than $1 \mathrm{MHz}$. The laser beam is split into a strong saturating beam and a weak probe beam that counterpropagate coaxially through the iodine cell. The beams in the cell are $2 \mathrm{~mm}$ in diameter and approximately collimated. The frequency of the saturating beam is shifted upward by $72 \mathrm{MHz}$ and its intensity is sinusoidally modulated at $85 \mathrm{kHz}$ by an acousto-optic modulator (AOM). The frequency shift introduced by the AOM prevents optical feedback from the counterpropagating beams into the dye laser. A variable attenuator provides for adjustment of the power in the saturating beam.

The probe beam passes through a travelling wave electro-optic phase modulator (EOM) that imposes symmetric sidebands about the laser center frequency. The sideband spacing is variable but is usually set to about the width of the transition to be observed. For $\mathrm{I}_{2}$ a spacing of 5 to $6 \mathrm{MHz}$ is used. The EOM drive power is adjusted to put $10 \%$ of the total laser power in each sideband by observing the spectrum of the probe beam on a $300 \mathrm{MHz}$ optical spectrum analyzer. As the laser frequency is scanned across the transition of interest the sidebands probe the narrow saturated absorption resonance induced by the saturating beam.

After passing through the $I_{2}$ cell a portion of the probe beam intensity is diverted by a beam splitter and focused on a fast photodiode. The resulting signal is processed by a high-pass filter, double-balanced mixer, and lockin amplifier to recover the anti-symmetric FM resonance (Figure 2). The phase of the local oscillator signal to the mixer is adjusted by a variable delay line to obtain an FM signal proportional to either the dispersion of the iodine hyperfine component 
(profile $S_{2}$ in Fig. 3 of Ref. 9) or its absorption (profile $S_{1}$ ). The dispersion profile is generally preferred because it has a steep zero crossing that is independent of the relative sizes of the line width and the sideband spacing and is very suitable for locking the laser to the iodine line. In some of our early FM measurements the absorption phase was used because at that time we could observe it with better signal-to-noise ratio.

The iodine cell used in this work was fabricated at NIST. The methods used in preparing the cell were identical to those used in making cells for $\mathrm{I}_{2}$ stabilized lasers constructed at NIST. 10 Previous tests with such cells have shown that frequency shifts due to residual background gas pressure are less than 1 part in $10^{11}$. The cell is $12 \mathrm{~mm}$ in diameter and $30 \mathrm{~cm}$ long with fused silica Brewster windows and a small sidearm. For all of our measurements the cell was operated in ambient laboratory air at a temperature of $23 \pm 1^{\circ} \mathrm{C}$. At this temperature our long cell and collinear beam geometry gave excellent signal-tonoise ratio even for transitions below $16000 \mathrm{~cm}^{-1}$ for which heated cells are sometimes required.

Lines for measurement were initially chosen by reference to the iodine atlas of Gerstenkorn and Luc. ${ }^{1}$ In general we tried to choose lines that were narrow and relatively isolated to reduce the likelihood of blended transitions and to make it easier to find the correct line for later use. The laser was tuned to the line of interest by using a travelling Michelson wavemeter with an accuracy of about $0.002 \mathrm{~cm}^{-1}$. For each line we first made a broadband scan (about $0.045 \mathrm{~cm}^{-1}$ wide) to observe the hyperfine structure and to identify a hyperfine component suitable for locking the dye laser. Lines with strong overlapping components from other transitions were normally rejected because of the difficulty of routinely identifying the correct component. 
After an appropriate hyperfine component had been selected, the dye laser was servo-locked to the zero crossing of its FM signal. The servo-lock was implemented by using the external scan input of the dye laser controller. It was able to maintain lock for an indefinite period, combining the long term stability of the $I_{2}$ resonance with the narrow line width of the commercial laser.

The absolute measurement of the dye laser wave number was made by using the Fabry-Perot wavemeter developed in our laboratory. ${ }^{11}$ This wavemeter has been used for numerous precise measurements and has demonstrated its ability to make reliable absolute measurements at a sub-MHz level of accuracy. Measurements with the wavemeter are made with respect to a $\mathrm{He}-\mathrm{Ne}$ laser stabilized to the $g$ component of the ${ }^{127} I_{2}$ transition $R(127) 11-5$. Our laser follows the same design as that used by Jennings et al. ${ }^{12}$ who determined the frequency of this line to be $473612340.492(74) \mathrm{MHz}$.

Almost all of the lines reported here were measured with both 218-mm and 7-mm Fabry-Perot spacers. Some lines were also measured with a 31-mm spacer. Results derived from observations with different spacer lengths were used to correct for phase dispersion on reflection from the coatings of the interferometer plates as previously described. ${ }^{13}$ The phase correction used in this work was calculated from a polynomial fit to all of our phase dispersion data from this and other experiments spanning the region 471 to $765 \mathrm{~nm}$. Measuring each line with spacers of different lengths also provides assurance that the correct integer order of interference has been used in calculating the wave number.

\section{RESULTS}

The results for all $I_{2}$ lines observed in this work are collected in Table 1 . For each line the Doppler-limited wave number as measured by Fourier 
transform spectroscopy is given in the first column. This value can be used to locate the line in the iodine atlas of Gerstenkorn and Luc. ${ }^{1}$

The line classifications given in the second column of Table 1 have been determined from calculations of the $\mathrm{I}_{2} \mathrm{~B}-\mathrm{X}$ band system made with Dunham parameters derived from the Fourier transform spectra. ${ }^{14}$ In general the $\mathrm{I}_{2}$ spectrum is very dense, with several transitions predicted within the Doppler profile of each line listed in the iodine atlas. The classification given in the table applies to the transition for which the resolved hyperfine component was measured. It is based on the wave number, hyperfine structure, and expected intensity of the line. Other transitions not listed may also contribute to the Doppler-broadened line. We found that three lines $\left(16339.7 \mathrm{~cm}^{-1}, 17016.1 \mathrm{~cm}^{-1}\right.$ and $17049.2 \mathrm{~cm}^{-1}$ ) are misidentified in Ref. 4. Corrected classifications for these lines are given in Table 1.

In the third column of Table 1 we list the hyperfine component that was measured for each line. There are 15 hyperfine components for lines with even $\mathrm{J}$ and 21 components for lines with odd $J$. This hyperfine structure was explained by Kroll ${ }^{15}$ as due primarily to the nuclear electric quadrupole interaction. Kroll points out that for high $\mathrm{J}$ the hyperfine components appear in six distinct groups as seen in Figs. 3 and 4. Most of our lines display this common pattern. For low $\mathrm{J}$, magnetic and second order electric quadrupole interactions become increasingly significant, and the distribution of components deviates from this pattern (Fig. 5).

In previous work there has been no standard convention for the labeling of the iodine hyperfine components. This has resulted in some confusion in their use. In this paper we use labels for the components as given by Gerstenkorn and Luc. ${ }^{2}$ This labeling is illustrated in Figs. 3 and 4. Note that for both 15- and 21- 
component lines the component labeled $o$ by Hlousek and Fairbank 4 corresponds to our component $t$.

For most lines we have chosen to measure the $t$ hyperfine component. This component is convenient because it is easy to locate even if one has no precise way of measuring the wavelength of a tunable laser. First, the correct iodine line is identified by comparing the Doppler-limited laser absorption (or fluorescence) spectrum with the spectrum in the iodine atlas. ${ }^{1}$ Then the laser is tuned onto the line from the low wave number side while observing the Doppler-free spectrum. For 15-component structures (Fig. 3) the $t$ component is the first one encountered; for 21-component structures (Fig. 4) it is the central component of the first group of three.

In the fourth column of the Table 1 we present the measured wave number of the specified component and its uncertainty. The value reported is the average of 2 to 9 independent measurements made on different days with the 218-mm Fabry-Perot spacer. Each of these independent measurements is the average of about 80 successive readings from the Fabry-Perot wavemeter taken over a period of about a minute.

The uncertainty at the one standard deviation level is derived as the quadrature sum of the standard error of the mean of the individual determinations (typically about $1 \times 10^{-5} \mathrm{~cm}^{-1}$ ), the estimated uncertainty of the lock point due to line asymmetry and baseline shifts $\left(6 \times 10^{-6} \mathrm{~cm}^{-1}\right)$, the uncertainty of the phase shift correction $\left(6 \times 10^{-6} \mathrm{~cm}^{-1}\right)$, the uncertainty of the reference laser wave number $\left(2.3 \times 10^{-6} \mathrm{~cm}^{-1}\right)$, and the uncertainty in the correction for the reference laser power and temperature $\left(2.8 \times 10^{-6} \mathrm{~cm}^{-1}\right)$. The uncertainty reported in Table 1 is two standard deviations and represents a $95 \%$ confidence level. 
To assist in identifying the correct line and component for each measured transition, descriptive comments are included in the final column of Table 1 . The first comment line describes the number and distribution of hyperfine components. In most cases the observed hyperfine components fall in six clearly defined groups. The code $\mathrm{W}$ is used to indicate the few lines with low $\mathbf{J}$ for which the components are fully resolved but distributed in such a way that these normal groupings are not apparent. For lines in which overlapping hyperfine components from other transitions are observed, additional comment lines are added that describe the interfering transitions. These comments can be understood by comparing the tabulated information for the line at $16254 \mathrm{~cm}^{-1}$ to the spectrum for this line which is reproduced in Fig. 5.

\section{DISCUSSION}

Our current results are compared with some previously reported measurements in Fig. 6. For clarity not all previous measurements are included. We have chosen to compare with results of Hlousek and Fairbank ${ }^{4}$ and of Shiner et al. ${ }^{6}$ because they have the most lines in common with this work. We also compare to three lines recommended by the Comité Consultatif pour la Définition du Mètre (CCDM). ${ }^{3}$ These three values represent the combined measurements of several laboratories and should be the most reliable absolute measurements in $I_{2}$.

The comparison in Fig. 6 shows excellent agreement between the various measurements. All values are consistent within their quoted uncertainties with the exception of the $17030-\mathrm{cm}^{-1}$ line. For this line it appears that the result of Hlousek and Fairbank 4 is in error. Difficulty with this line is confirmed by experimental work at the Free University of Amsterdam, where the value of Hlousek and Fairbank was found to be inconsistent with other reference lines. ${ }^{16}$ 
It is apparent from Fig. 6 that the wave numbers measured in the present work are slightly smaller than those measured in other laboratories. With respect to the CCDM recommended values, this offset is nearly constant at an average of $-210 \pm 30 \mathrm{kHz}$. The offset from Shiner et al. has a greater scatter and averages $-277 \pm 146 \mathrm{kHz}$. These differences are much smaller than our estimated uncertainty but are nevertheless of some concern.

To investigate this problem we have compared the frequency of our $\mathrm{I}_{2}$ stabilized reference laser with a second $\mathrm{I}_{2}$-stabilized laser. The frequency of the laser used for our present measurements was found to be slightly higher than that of the comparison laser. In four determinations with realignment of the laser cavity the offset ranged from 14 to $45 \mathrm{kHz}$. This is a relative difference of about 9 parts in $10^{-11}$, well within the reported uncertainty of the reference laser frequency and in the wrong direction to explain our difference with other laboratories. We have also done tests in which we measured the same iodine line using different $\mathrm{AOM}$ frequencies, locking the reference laser to different iodine components, and inverting the phase in the FM detection electronics. None of these tests showed a systematic effect that might contribute to the observed difference.

In Fig. 7 our present results are compared to the 19 lines measured with lower precision by Hlousek and Fairbank. 4 Again the agreement is satisfactory. Although the measurements seem to diverge with increasing wave number, all of the values agree well within the $6 \mathrm{MHz}$ uncertainty of Ref. 4. For these lines our present results are more accurate than the previous values by a factor of about 6 .

In summary we have established accurate reference wave numbers for 102 Doppler-free hyperfine components in ${ }^{127} \mathrm{I}_{2}$. These lines cover the range from 560 to $656 \mathrm{~nm}$ (15233 to $17845 \mathrm{~cm}^{-1}$ ) with no gaps wider than $50 \mathrm{~cm}^{-1}$. For most of the 
lines the absolute accuracy is better than $1 \mathrm{MHz}$ (95\% confidence interval). These results should be useful as reference lines for laser spectroscopy and for calibration of wavemeters and interferometers.

\section{ACKNOWLEDGMENTS}

The help of Kevin J. Coakley of the NIST Statistical Engineering Division, who analyzed our phase dispersion data by using a statistical bootstrap procedure to obtain a globally optimized phase correction function, is gratefully acknowledged. The author also wishes to thank Howard Layer for filling the iodine cell used in this work and John Gillaspy for helpful discussions concerning Doppler-free FM spectroscopy.

Portions of this work were sponsored by Lawrence Livermore National Laboratory under contract \#923-003. 


\section{References}

1. S. Gerstenkorn and P. Luc, "Atlas du Spectra D'Absorption de la Molecule D'Iode," (editions du Centre National de la Research Scientifique, 15 quai Anatole-France, 75700 Paris, 1978).

2. S. Gerstenkorn and P. Luc, "Absolute iodine $\left(I_{2}\right)$ standards measured by means of Fourier transform spectroscopy," Revue Phys. App. 14, 791-794 (1979).

3. T. J. Quinn, "Mise en Pratique of the Definition of the Metre (1992)," Metrologia 30, 523-541 (1993).

4. L. Hlousek and W. M. Fairbank, Jr., "High-accuracy wave-number measurements in molecular iodine," Opt. Lett. 8, 322-323 (1983).

5. P. Juncar, J. Pinard, J. Hamon, and A. Chartier, "Absolute determination of the Wavelengths of the Sodium $\mathrm{D}_{1}$ and $\mathrm{D}_{2}$ Lines by Using a $\mathrm{CW}$ Tunable Dye Laser Stabilized on Iodine," Metrologia 17, 77-79 (1981).

6. D. Shiner, J. M. Gilligan, B. M. Cook, and W. Lichten, " $\mathrm{H}_{2}, \mathrm{D}_{2}$, and HD ionization potentials by accurate calibration of several iodine lines," Phys. Rev. A 47, 4042-4045 (1993).

7. R. Grieser, G. Bönsch, S. Dickopf, G. Huber, R. Klein, P. Merz, A. Nicolaus, and H. Schnatz, "Precision measurement of two iodine lines at $585 \mathrm{~nm}$ and 549 nm ," Z. für Phys. A 342, 147-150 (1994). 
8. J. L. Hall, L. Hollberg, T. Baer, and H. G. Robinson, "Optical heterodyne saturation spectroscopy," Appl. Phys. Lett. 39, 680-682 (1981).

9. G. C. Bjorklund, M. D. Levenson, W. Lenth, and C. Ortiz, "Frequency Modulation (FM) Spectroscopy," Appl. Phys. B 32, 145-152 (1983).

10. H. P. Layer, "A Portable Iodine Stabilized Helium-Neon Laser, " IEEE Trans. Inst. Meas. IM-29, 358-361 (1980).

11. C. J. Sansonetti, in Advances in Laser Science - IV, edited by J. L. Gole, D. F. Heller, M. Lapp, and W. C. Stwalley (AIP, New York, 1988), pp. 548-553.

12. D. A. Jennings, C. R. Pollock, F. R. Petersen, R. E. Drullinger, K. M. Evenson, J. S. Wells, J. L. Hall, and H. P. Layer, "Direct frequency measurement of the $\mathrm{I}_{2}$-stabilized He-Ne 473-THz (633-nm) laser," Opt. Lett. 8, 136-138 (1983).

13. J. D. Gillaspy and C. J. Sansonetti, "Absolute wavelength determinations in molecular tellurium: new reference lines for precision laser spectroscopy," J. Opt. Soc. Am. B 8, 2414-2419 (1991).

14. S. Gerstenkorn and P. Luc, "Description of the absorption spectrum of iodine recorded by means of Fourier Transform Spectroscopy: the (B-X) system," J. Physique 46, 867-881 (1985).

15. M. Kroll, "Hyperfine Structure of the Visible Molecular-Iodine Absorption Spectrum," Phys. Rev. Lett. 23, 631-633 (1969). 
16. W. Ubachs, private communication (1995). 


\section{Figure Captions}

Fig. 1. Experimental apparatus for observation of Doppler-free absorption lines in molecular iodine.

Fig. 2. Signal processing schematic for FM spectroscopy and laser locking to iodine resonances.

Fig. 3. Spectrum of the transition $P 80(15-2)$ at $16917 \mathrm{~cm}^{-1}$, a typical 15 component hyperfine structure.

Fig. 4. Spectrum of the transition R47 (9-3) at $16130 \mathrm{~cm}^{-1}$, a typical 21 component hyperfine structure.

Fig. 5. Overlapping hyperfine structures at $16254 \mathrm{~cm}^{-1}$ which illustrate the codes used in the comments of Table 1.

Fig. 6. Deviations of previous precise measurements from the present results (previous measurement - this work). The shaded area for each line represents the uncertainty of the present results.

Fig.7. Deviations of the lines measured with lower precision by Hlousek and Fairbank 4 from the present results. The uncertainty of of Hlousek and Fairbank is $6 \mathrm{MHz}$. The typical uncertainty for the present work is $1 \mathrm{MHz}$, indicated by the shaded region in the figure. 
Table 1. Measured Wave Numbers of Hyperfine Components in $I_{2}$.

\begin{tabular}{|c|c|c|c|c|}
\hline $\begin{array}{l}\text { Atlas Wave } \\
\text { Numbera } \\
\left(\mathrm{cm}^{-1}\right)\end{array}$ & Transition & Component & $\begin{array}{c}\text { Measured } \\
\text { Wave Number } \\
\left(\mathrm{cm}^{-1}\right)\end{array}$ & Commentsc \\
\hline 15233.3653 & $\mathrm{R} 73(5-5)$ & $i$ & $15233.367441(21)$ & $21 \cup(p, q)$ \\
\hline 15278.5914 & $\mathrm{P} 110(6-5)$ & $t$ & $15278.574318(20)$ & $15 \cup(d, e)(f, g)(p, q)$ \\
\hline 15297.7275 & $\mathrm{P} 100(6-5)$ & $t$ & $15297.712483(24)$ & $\begin{array}{l}15 \mathrm{U}(d, e)(f, g)(p, q) \\
\mathrm{P} 149(7-5)-790 \mathrm{MHz} 15 \%\end{array}$ \\
\hline 15341.8700 & P72 (6-5) & $t$ & $15341.853034(23)$ & $15 \mathrm{U}(d, e)(f, g)$ \\
\hline 15379.2371 & $\mathrm{R} 41(6-5)$ & $t$ & $15379.221566(21)$ & $\begin{array}{l}21 \mathrm{R} \\
\mathrm{R} 75(10-7)-70 \mathrm{MHz} 3 \%\end{array}$ \\
\hline 15406.9515 & $\mathrm{P} 102(7-5)$ & $t$ & $15406.937545(27)$ & $\begin{array}{l}15 \mathrm{U}(d, e)(f, g)(p, q) \\
R 50(10-7)+560 \mathrm{MHz} 5 \%\end{array}$ \\
\hline 15411.4059 & R94 (5-4) & $t$ & $15411.389346(27)$ & $15 \cup(d, e)(f, g)(p, q)$ \\
\hline 15427.3234 & P77 (5-4) & $t$ & $15427.306474(22)$ & $21 \mathrm{U}(p, q)$ \\
\hline 15476.0688 & P55 (7-5) & $t$ & $15476.051750(24)$ & $21 R$ \\
\hline 15524.1299 & P89 (6-4) & $t$ & $15524.112357(23)$ & $21 U(p, q)$ \\
\hline 15575.1479 & $\mathrm{R} 56(6-4)$ & $t$ & $15575.137259(21)$ & $15 \mathrm{U}(d, e)(f, g)$ \\
\hline
\end{tabular}




$\begin{array}{rllll}15627.0467 & \mathrm{P} 95(7-4) & t & 15627.030692(25) & 21 \mathrm{U}(p, q) \\ 15672.5341 & \mathrm{P} 65(7-4) & t & 15672.517398(22) & 21 \mathrm{R} \\ 15727.5394 & \mathrm{R} 22(9-5) & t & 15727.518573(23) & 15 \mathrm{U}(f, g)(o, p) \\ 15758.9686 & \mathrm{R} 89(8-4) & t & 15758.949917(19) & 21 \mathrm{P}(p, q) \\ -- & \mathrm{P} 33(6-3) & r & 15797.967821(23) & 21 \mathrm{R} \\ 15807.2941 & \mathrm{R} 50(8-4) & t & 15807.267682(20) & 15 \mathrm{U}(d, e)(f, g) \\ & & & \mathrm{P} 44(8-4)+410 \mathrm{MHz} 70 \% \\ 15846.3960 & & & & \mathrm{P} 115(9-4)+520 \mathrm{MHz} 25 \% \\ 15856.9424 & \mathrm{P} 96(9-4) & t & 15846.384979(22) & \mathrm{R} 20(6-3)+760 \mathrm{MHz} 10 \% \\ 15886.5111 & \mathrm{R} 96(9-4) & t & 15856.931277(20) & 15 \mathrm{U}(d, e)(f, g)(p, q) \\ 15919.8992 & \mathrm{P} 61(7-3) & t & 15886.494949(24) & 21 \mathrm{R}(d, e)(f, g)(p, q) \\ 15954.0339 & \mathrm{R} 48(9-4) & t & 15919.884050(23) & 15 \mathrm{U}(d, e)(f, g) \\ & \mathrm{R} 97(8-3) & t & 15954.016985(24) & 21 \mathrm{U}(p, q) \\ 15973.6499 & & & & \mathrm{R} 146(11-4)-160 \mathrm{MHz} 15 \% \\ 16038.2085 & \mathrm{R} 91(10-4) & t & 15973.631183(30) & 21 \mathrm{U}(p, q) \\ 16052.4447 & \mathrm{R} 36(10-4) & t & 16038.192673(21) & 15 \mathrm{U}(d, e)(f, g) \\ & \mathrm{R} 103(9-3) & t & 16052.429625(19) & 21 \mathrm{U}(p, q) \\ & & & & \mathrm{R} 97(7-2)-200 \mathrm{MHz} 10 \%\end{array}$




\begin{tabular}{|c|c|c|c|c|}
\hline 16090.5320 & P75 (9-3) & $t$ & $16090.515101(25)$ & $21 \mathrm{R}$ \\
\hline 16130.0815 & R47 (9-3) & $\boldsymbol{t}$ & $16130.062709(23)$ & $21 \mathrm{R}$ \\
\hline 16172.3266 & P91 (10-3) & $t$ & $16172.309240(23)$ & $21 \mathrm{U}(p, q)$ \\
\hline \multirow[t]{2}{*}{16205.9611} & R76 (10-3) & $t$ & $16205.946017(20)$ & $15 \mathrm{U}(d, e)(f, g)(p, q)$ \\
\hline & & & & R78 (12-4) -650 MHz 20\% \\
\hline 16254.6514 & P17 (10-3) & $t$ & $16254.623391(23)$ & $\begin{array}{l}21 \mathrm{~W} \\
\text { P26 (12-4) +240 MHz 20\% }\end{array}$ \\
\hline 16304.5153 & P76 (11-3) & $t$ & $16304.499027(31)$ & $15 \mathrm{U}(d, e)(f, g)(p, q)$ \\
\hline 16339.7841 & R48 (9-2) & $t$ & $16339.768615(36)$ & $15 \mathrm{U}(d, e)(f, g)$ \\
\hline 16340.6383 & $\mathrm{R} 47(9-2)$ & $i$ & $16340.658054(31)$ & $\begin{array}{l}21 \mathrm{R} \\
\mathrm{P} 48(11-3)-700 \mathrm{MHz} 170 \%\end{array}$ \\
\hline 16341.7580 & $\mathrm{R} 40(9-2)$ & $t$ & $16341.742422(20)$ & $15 \mathrm{U}(d, e)(f, g)(o, p)(q, r)$ \\
\hline 16394.6975 & P84 (10-2) & $t$ & $16394.681360(27)$ & $15 \cup(d, e)(f, g)(p, q)$ \\
\hline 16404.6432 & P78 (10-2) & $t$ & $16404.626597(30)$ & $15 \mathrm{U}(d, e)(f, g)(p, q)$ \\
\hline 16424.4665 & $\mathrm{R} 70(10-2)$ & $t$ & $16424.450522(23)$ & $15 \mathrm{U}(d, e)(f, g)(p, q)$ \\
\hline \multirow[t]{2}{*}{16452.4119} & R44 (10-2) & $t$ & $16452.395097(23)$ & $15 \mathrm{U}(d, e)(f, g)$ \\
\hline & & & & $\mathrm{P} 176(13-2)+540 \mathrm{MHz} 5 \%$ \\
\hline \multirow[t]{2}{*}{16502.8340} & P82 (13-3) & $t$ & $16502.817062(21)$ & $15 \cup(d, e)(f, g)(p, q)$ \\
\hline & & & & $P 69(17-5)+4507 \%$ \\
\hline 16516.8633 & $\mathrm{R} 80(11-2)$ & $t$ & $16516.846551(24)$ & $15 \mathrm{U}(d, e)(f, g)(p, q)$ \\
\hline
\end{tabular}




$\begin{array}{lllll}16568.2732 & \mathrm{P} 27(11-2) & t & 16568.255050(23) & 21 \mathrm{~W} \\ 16569.9911 & & & & \mathrm{P} 16(9-1)+320 \mathrm{MHz} 4 \% \\ 16624.9391 & \mathrm{P} 24(11-2) & t & 16569.974576(25) & 15 \mathrm{U}(d, e)(f, g)(o, p)(q, r) \\ 16638.0457 & \mathrm{R} 78(12-2) & t & 16624.923159(25) & 15 \mathrm{U}(d, e)(f, g)(p, q) \\ & \mathrm{R} 69(12-2) & i & 16638.049703(23) & 21 \mathrm{U}(p, q) \\ & & & \mathrm{P} 117(13-2)+68 \mathrm{MHz} 45 \% \\ 16686.0552 & \mathrm{P} 96(13-2) & t & 16686.039067(21) & \mathrm{R} 156(14-2)-590 \mathrm{MHz} 20 \% \\ 16745.5928 & \mathrm{R} 66(13-2) & t & 16745.576624(30) & 15 \mathrm{U}(d, e)(f, g)(p, q) \\ 16762.1363 & \mathrm{R} 52(13-2) & t & 16762.120059(21) & 15 \mathrm{U}(d, e)(f, g) \\ 16780.8572 & \mathrm{P} 26(11-1) & t & 16780.840058(24) & 15 \mathrm{U}(d, e)(f, g)(o, p)(q, r) \\ & & & & \mathrm{R} 169(16-2)+730 \mathrm{MHz} 50 \% \\ 16806.6446 & \mathrm{R} 95(12-1) & t & 16806.627895(28) & 21 \mathrm{U}(p, q) \\ & & & & \mathrm{P} 187(15-1)+620 \mathrm{MHz} 7 \% \\ 16833.1260 & \mathrm{R} 8(12-1) & t & 16833.109663(46) & 15 \mathrm{U}(d, e)(f, g)(p, q) \\ 16875.1260 & \mathrm{P} 106(13-1) & t & 16875.109221(26) & 15 \mathrm{U}(d, e)(f, g)(p, q) \\ 16891.0383 & \mathrm{P} 18(12-1) & t & 16891.022622(35) & 15 \mathrm{U}(f, g)(o, p) \\ 16917.3476 & \mathrm{P} 80(15-2) & t & 16917.332049(20) & 15 \mathrm{U}(d, e)(f, g)(p, q) \\ 16951.6035 & \mathrm{R} 70(13-1) & t & 16951.597697(54) & 15 \mathrm{U}(d, e)(f, g)(p, q) \\ & & & & \mathrm{R} 103(18-3)-6801 \% \\ & & & & \end{array}$




\begin{tabular}{|c|c|c|c|c|}
\hline 17006.0615 & R97 (14-1) & $t$ & $17006.044184(24)$ & $21 \cup(p, q)$ \\
\hline \multirow[t]{3}{*}{17015.0613} & P80 (16-2) & $t$ & $17015.045402(22)$ & $15 U(d, e)(f, g)(p, q)$ \\
\hline & & & & P50 (20-4) $-40 \mathrm{MHz} \quad 10 \%$ \\
\hline & & & & P6 (22-4) -1100 MHz 2\% \\
\hline 17016.1395 & P87 (14-1) & $t$ & $17016.122831(19)$ & $21 \cup(p, q)$ \\
\hline 17016.8724 & $\mathrm{P} 79(16-2)$ & $t$ & $17016.856207(19)$ & $21 \cup(p, q)$ \\
\hline 17018.0566 & P86 (14-1) & $t$ & $17018.039997(31)$ & $15 U(d, e)(f, g)(p, q)$ \\
\hline \multirow[t]{3}{*}{17018.6605} & P78 (16-2) & $t$ & $17018.644771(20)$ & $15 \mathrm{U}(d, e)(f, g)(p, q)$ \\
\hline & & & & $\mathrm{P} 90(12-0)-60 \mathrm{MHz} 25 \%$ \\
\hline & & & & P47 (20-4) $-1360 \mathrm{MHz} 25 \%$ \\
\hline 17021.8254 & P84 (14-1) & $t$ & $17021.809376(19)$ & $15 \mathrm{U}(d, e)(f, g)(p, q)$ \\
\hline 17026.8345 & R78 (16-2) & $t$ & $17026.818913(28)$ & $15 \mathrm{U}(d, e)(f, g)(p, q)$ \\
\hline 17029.1037 & P80 (14-1) & $t$ & $17029.088451(19)$ & $15 U(d, e)(f, g)(p, q)$ \\
\hline 17030.4320 & P123 (15-1) & $t$ & $17030.415189(25)$ & $21 \cup(p, q)$ \\
\hline 17038.3948 & P66 (16-2) & $t$ & $17038.378860(33)$ & $15 \mathrm{U}(d, e)(f, g)(p, q)$ \\
\hline \multirow[t]{2}{*}{17045.9627} & $\mathrm{R} 122(15-1)$ & $t$ & $17045.945455(23)$ & $15 \cdot \mathrm{U}(d, e)(f, g)(p, q)$ \\
\hline & & & & $\mathrm{P} 85(21-4)+480 \mathrm{MHz} 15 \%$ \\
\hline 17048.0993 & $\mathrm{R} 64(16-2)$ & $t$ & $17048.083923(25)$ & $15 U(d, e)(f, g)(p, q)$ \\
\hline 17049.2328 & P116(15-1) & $t$ & $17049.217506(53)$ & $15 \mathrm{U}(d, e)(f, g)(p, q)$ \\
\hline 17099.3577 & P86 (17-2) & $t$ & $17099.342383(20)$ & $15 \cup(d, e)(f, g)(p, q)$ \\
\hline
\end{tabular}




\begin{tabular}{|c|c|c|c|c|}
\hline 17101.1754 & P94 (15-1) & $t$ & $17101.180948(21)$ & $15 \mathrm{U}(d, e)(f, g)(p, q)$ \\
\hline \multirow[t]{2}{*}{17123.3262} & P73 (17-2) & $t$ & $17123.309988(29)$ & $21 \cup(p, q)$ \\
\hline & & & & P31 (21-4) +550 MHz 15\% \\
\hline \multirow[t]{3}{*}{17144.3553} & $R 64(17-2)$ & $t$ & $17144.338765(27)$ & $15 \cup(d, e)(f, g)(p, q)$ \\
\hline & & & & R83 (22-4) -980 MHz 5\% \\
\hline & & & & P171 (18-1) -1100 MHz 5\% \\
\hline 17157.4114 & R67 (15-1) & $t$ & $17157.396892(28)$ & $21 \cup(p, q)$ \\
\hline \multirow[t]{2}{*}{17166.0191} & P108 (16-1) & $t$ & $17166.002813(23)$ & $15 \mathrm{U}(d, e)(f, g)(p, q)$ \\
\hline & & & & P67 (32-4) +90 MHz 5\% \\
\hline 17194.9067 & $\mathrm{R} 30(15-1)$ & $t$ & $17194.891763(29)$ & $15 \cup(d, e)(f, g)$ \\
\hline 17199.8446 & $\mathrm{R} 98(16-1)$ & $t$ & $17199.827846(21)$ & $15 \mathrm{U}(d, e)(f, g)(p, q)$ \\
\hline \multirow[t]{2}{*}{17205.8047} & P98 (14-0) & $t$ & $17205.788933(28)$ & $15 \mathrm{U}(d, e)(f, g)(p, q)$ \\
\hline & & & & $\mathrm{R} 140(15-0)-60 \mathrm{MHz} 40 \%$ \\
\hline \multirow[t]{2}{*}{17252.0411} & P74 (14-0) & $t$ & $17252.023212(21)$ & $15 \mathrm{U}(d, e)(f, g)(p, q)$ \\
\hline & & & & $\mathrm{R} 105(19-2)+160 \mathrm{MHz} 50 \%$ \\
\hline \multirow[t]{2}{*}{17300.0056} & $\mathrm{P} 12(16-2)$ & $t$ & $17299.991091(48)$ & $15 \mathrm{R}$ \\
\hline & & & & $\mathrm{R} 168(22-2)-550 \mathrm{MHz} 2 \%$ \\
\hline 17349.6773 & $\mathrm{R} 80(15-0)$ & $t$ & $17349.661322(24)$ & $15 U(d, e)(f, g)(p, q)$ \\
\hline 17352.2451 & P62 (17-1) & $t$ & $17352.231328(28)$ & $15 \cup(d, e)(f, g)(p, q)$ \\
\hline
\end{tabular}




$\begin{array}{lllll}17360.6176 & \mathrm{P} 56(17-1) & t & 17360.601438(34) & 15 \mathrm{U}(d, e)(f, g) \\ 17380.8317 & \mathrm{P} 38(17-1) & t & 17380.816923(21) & 15 \mathrm{P}(d, e)(f, g) \\ 17405.1703 & \mathrm{R} 90(18-1) & t & 17405.154995(31) & 15 \mathrm{U}(d, e)(f, g)(p, q) \mathrm{d} \\ 17448.3784 & \mathrm{R} 42(20-2) & t & 17448.363077(24) & 15 \mathrm{U}(d, e)(f, g) \\ 17450.7924 & \mathrm{R} 78(16-0) & t & 17450.775435(39) & 15 \mathrm{U}(d, e)(f, g)(p, q) \\ 17461.1972 & \mathrm{P} 67(16-0) & t & 17461.182208(29) & 21 \mathrm{U}(p, q) \\ 17500.8423 & \mathrm{R} 88(19-1) & t & 17500.827932(42) & 15 \mathrm{U}(d, e)(f, g)(p, q) \\ 17501.1370 & \mathrm{R} 38(16-0) & t & 17501.121870(26) & 15 \mathrm{U}(d, e)(f, g) \\ & & & & \mathrm{P} 70(29-5)-500 \mathrm{MHz} 15 \% \\ 17521.7500 & \mathrm{R} 77(19-1) & t & 17521.737626(26) & 21 \mathrm{U}(p, q) \\ 17531.3938 & \mathrm{P} 67(19-1) & t & 17531.384093(22) & 21 \mathrm{U}(p, q) \\ 17550.0663 & \mathrm{R} 76(17-0) & t & 17550.050483(53) & 15 \mathrm{U}(d, e)(f, g)(p, q) \\ & & & & \mathrm{P} 125(26-3)-350 \mathrm{MHz} 15 \% \\ 17578.5963 & \mathrm{P} 26(19-1) & i & 17578.599454(22) & 15 \mathrm{U}(d, e)(f, g) \\ 17605.3257 & & & & \mathrm{R} 92(25-3)-840 \mathrm{MHz} 30 \% \\ 17609.8910 & \mathrm{P} 23(17-0) & t & 17605.308074(29) & 21 \mathrm{~W} \\ 17655.9473 & \mathrm{R} 18(17-0) & t & 17609.875064(36) & 15 \mathrm{P}(f, g)(q, r) \\ & & & & \mathrm{P} 159(23-1) ?+430 \mathrm{MHz} 30 \% \\ & \mathrm{P} 42(20-1) & t & 17655.932802(41) & 15 \mathrm{U}(d, e)(f, g)\end{array}$




$\begin{array}{lllll}17668.6723 & \mathrm{R} 32(20-1) & t & 17668.657200(29) & 15 \mathrm{U}(d, e)(f, g) \\ 17685.4130 & \mathrm{R} 46(18-0) & t & 17685.398042(22) & 15 \mathrm{U}(d, e)(f, g) \\ 17704.6867 & \mathrm{R} 19(18-0) & t & 17704.669058(20) & 21 \mathrm{~W} \\ 17742.5173 & \mathrm{R} 68(19-0) & t & 17742.501454(22) & 15 \mathrm{U}(d, e)(f, g)(p, q) \\ 17811.1027 & \mathrm{R} 84(20-0) & t & 17811.087494(21) & 15 \mathrm{U}(d, e)(f, g)(p, q) \\ & & & & \mathrm{R} 49(27-3)-100 \mathrm{MHz} 20 \% \\ 17845.2272 & \mathrm{P} 28(22-1) & t & 17845.202199(20) & 15 \mathrm{R} \\ & & & & \mathrm{R} 32(22-1)+390 \mathrm{MHz} 100 \%\end{array}$

a Ref. 1 with correction of Ref. 2.

b The uncertainty in parentheses represents a $95 \%$ confidence interval.

c The first comment line contains:

- The number of hyperfine components.

- A code describing the hyperfine structure as follows:

$$
\begin{aligned}
& \mathrm{R}=\text { fully resolved } \\
& \mathrm{U}=\text { not all components resolved } \\
& \mathrm{W}=\text { widely distributed structure without } 6 \text { clear groups of components. }
\end{aligned}
$$

- A list of pairs of components that were not resolved under our experimental conditions.

Additional comment lines are present only if components of another transition were observed in the same laser scan with the transition of interest. Each additional line contains: 
- The classification of the overlapping or adjacent transition.

- An estimate of the offset between the centers of gravity of the transition of interest and the overlapping transition.

- A rough estimate of the strength of the overlapping transition as a percentage of the transition of interest.

d Recorded scan not available for this transition. Calculations show no adjacent or overlapping lines. Inspection of other transitions in this band indicate that these are the likely unresolved components. 


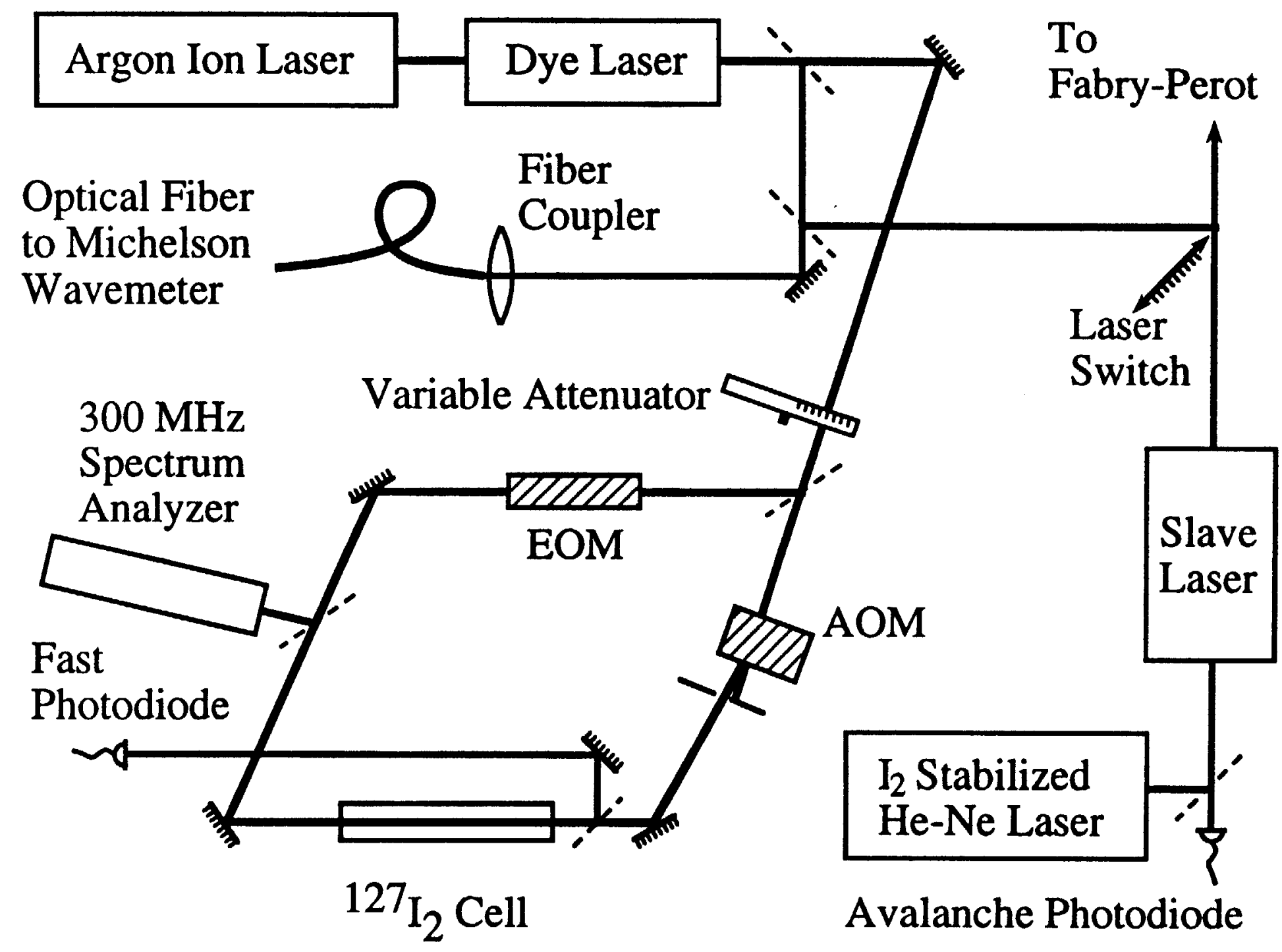




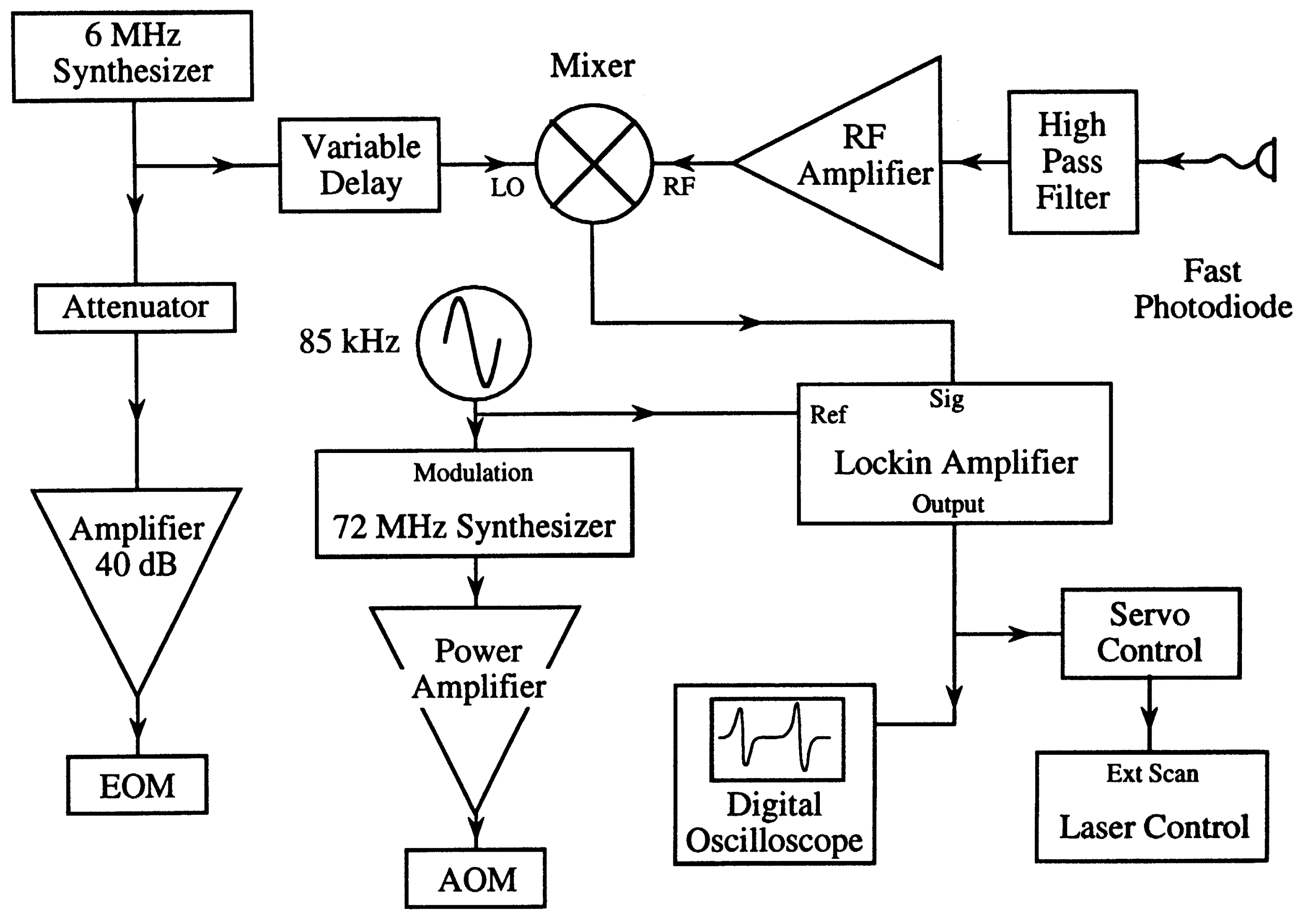

$F_{i,} 2$ 


$$
\text { Hilif }
$$




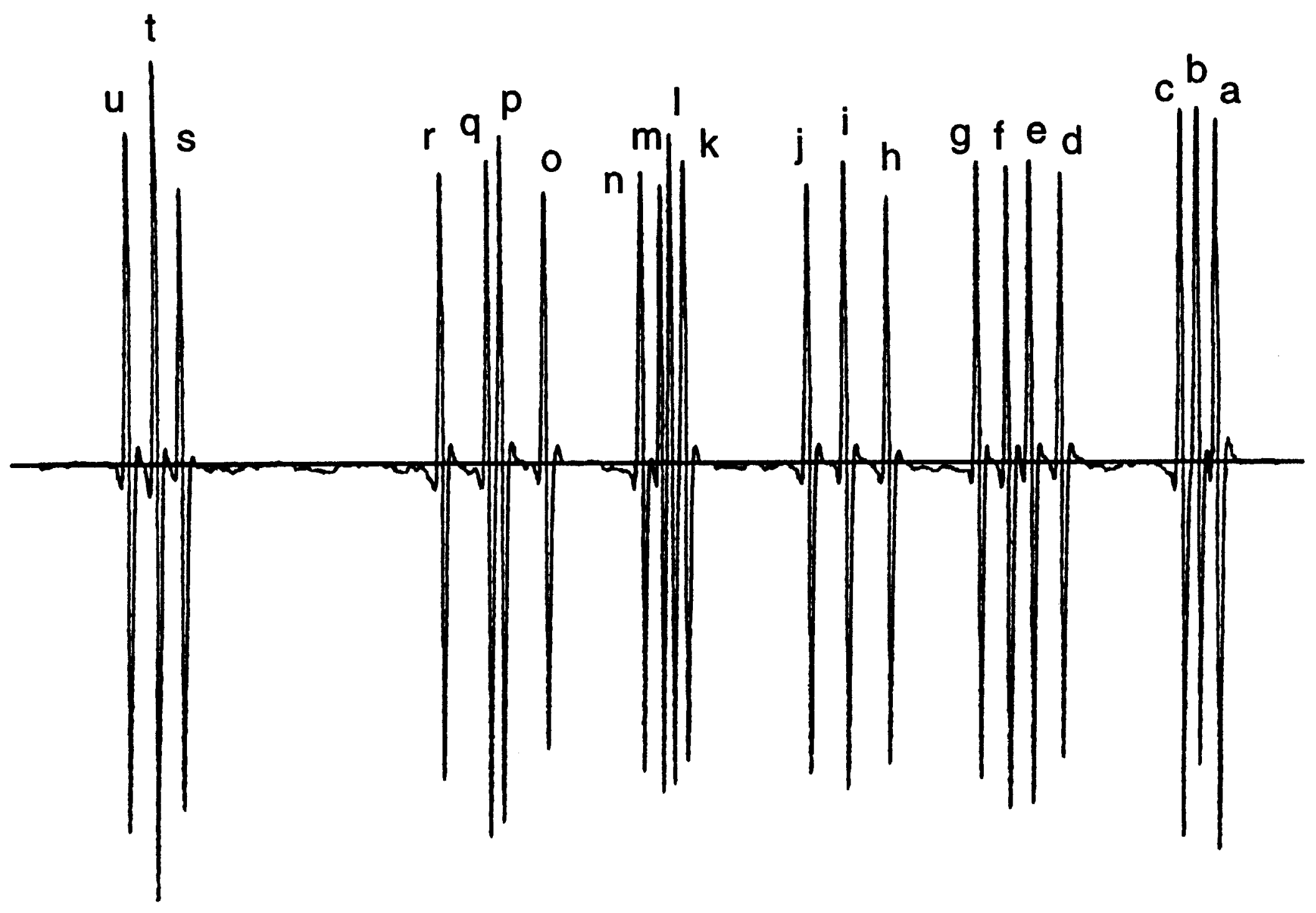




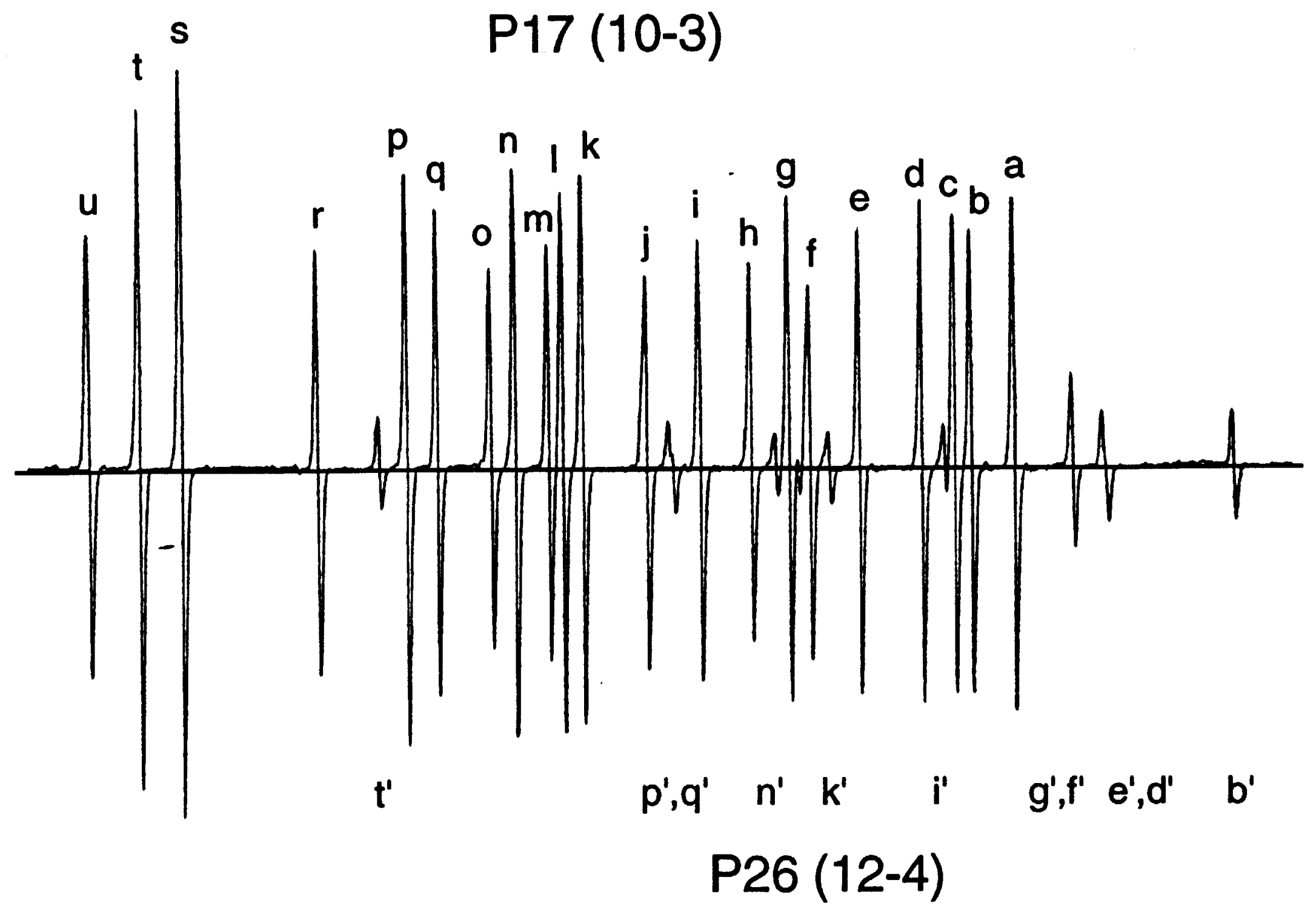




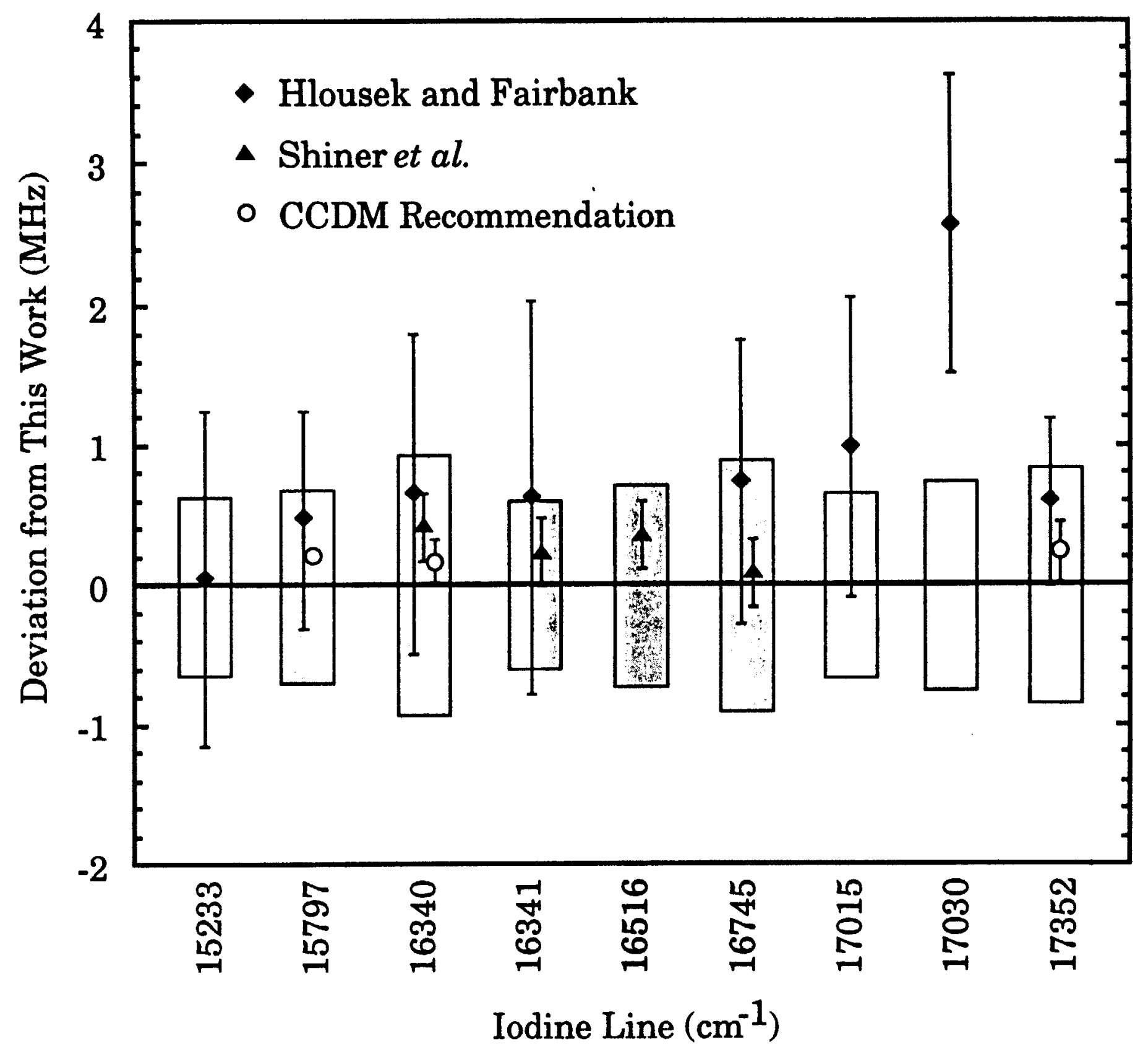




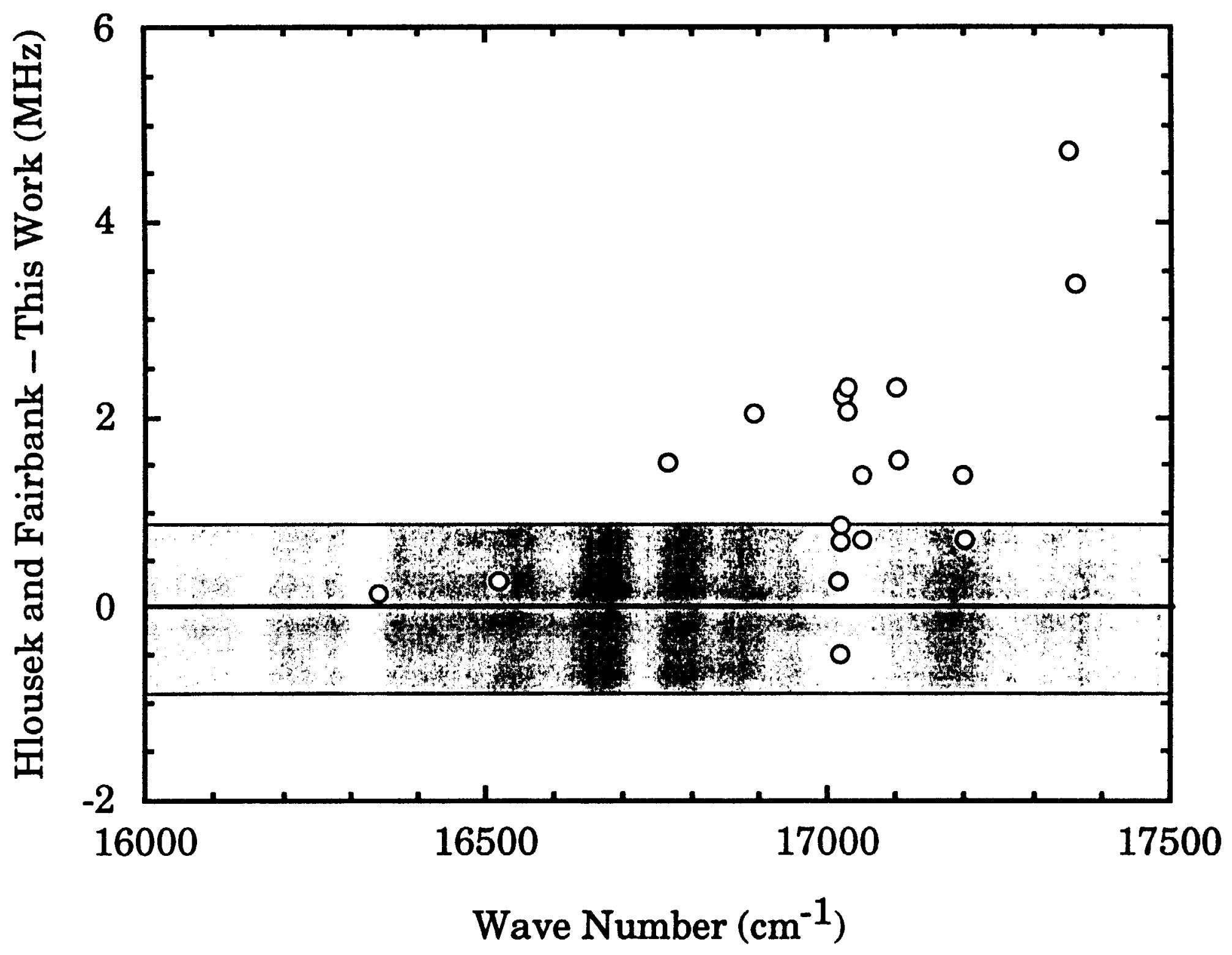




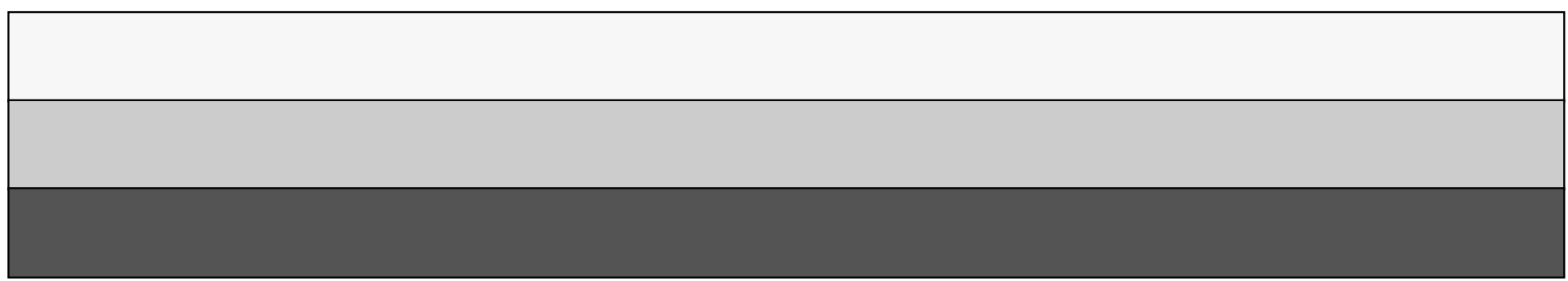

